\title{
A periodic linear-quadratic controller for suppressing rotor-blade vibration
}

\author{
Camino, J. F.; Santos, I. F.
}

Published in:

Journal of Vibration and Control

Link to article, DOI:

10.1177/1077546319853358

Publication date:

2019

Document Version

Early version, also known as pre-print

Link back to DTU Orbit

Citation (APA):

Camino, J. F., \& Santos, I. F. (2019). A periodic linear-quadratic controller for suppressing rotor-blade vibration. Journal of Vibration and Control, 25(17), 2351-2364. https://doi.org/10.1177/1077546319853358

\section{General rights}

Copyright and moral rights for the publications made accessible in the public portal are retained by the authors and/or other copyright owners and it is a condition of accessing publications that users recognise and abide by the legal requirements associated with these rights.

- Users may download and print one copy of any publication from the public portal for the purpose of private study or research.

- You may not further distribute the material or use it for any profit-making activity or commercial gain

- You may freely distribute the URL identifying the publication in the public portal

If you believe that this document breaches copyright please contact us providing details, and we will remove access to the work immediately and investigate your claim. 


\title{
A periodic linear quadratic controller for suppressing rotor-blade vibration
}

\author{
J. F. Camino' ${ }^{1}$ I. F. Santos ${ }^{2}$
}

\begin{abstract}
This paper presents an active control strategy, based on a time-varying linear quadratic optimal control problem, to attenuate the tip vibration of a two-dimensional coupled rotor-blade system whose dynamics is periodic. First, a periodic full-state feedback controller based on the linear quadratic regulator (LQR) problem is designed. If all the states are not available for feedback, then an optimal periodic time-varying estimator, using the Kalman-Bucy filter, is computed. Both the Kalman filter gain and the LQR gain are obtained as the solution of a periodic Riccati differential equation (PRDE). Together, these gains provide the observer-based LQG controller. An algorithm to solve the PRDE is also presented. Both controller designs ensure closed-loop stability and performance for the linear time-varying rotor-blade equation of motion. Numerical simulations show that the LQR and the LQG controllers are able to significantly attenuate rotor-blade tip vibration.
\end{abstract}

\section{Keywords}

Rotor dynamics, vibration control, periodic systems, linear time-varying (LTV) systems, LQG controller.

\section{Introduction}

Many engineering applications have rotor-blade systems as their main component, for which active control has been an attractive approach to improve performance, suppress vibrations, and prolong machinery lifetime (Firoozian and Stanway 1988; Khulief 2001; Szász and Flowers 2000), since passive damping methods are not able to attain higher efficiency. Over the last decades, many control design techniques for rotor-blade systems have been reported (Arcara et al. 2000; Sinha and Joseph 1994; Szász and Flowers 2001; Christensen and Santos 2005, and references therein).

It has been shown in Szász and Flowers (2001) that rotor and blade vibrations can be controlled by a shaftbased actuation if the rotor blades are deliberately mistuned. In Christensen (2004), a proportional-derivative feedback law is applied. An experimental contribution is given in Christensen and Santos (2005), which shows the feasibility of a periodic modal control strategy. However, due to the periodic time-varying nature of the problem, applications of classical LTI analysis and synthesis techniques are not suitable.

Most of the results available in the literature on active vibration control of rotor-blade systems (Khulief 2001; Szász and Flowers 2001, 2000; Fitzgerald et al. 2018, and references therein) are, in general, not able to guarantee either closed-loop stability or performance specifications whenever all the matrices of the rotor-blade equation of motion are time-varying. Even works that rely on the Floquet-Lyapunov decomposition (Christensen and Santos
2005, 2006; Skjoldan and Hansen 2009; Sinha and Joseph 1994; Wiedemann and Person 1992; Sherrill et al. 2015) to transform the time-variant system-dynamics matrix into a time-invariant matrix still suffer from this drawback, since the input and output matrices in the state-space representation remain time-variant and, consequently, the direct application of linear time-invariant (LTI) control design techniques is not suitable.

In order to guarantee closed-loop stability and performance for the rotor-blade system, a time-varying optimal linear quadratic Gaussian (LQG) control design is proposed, which allows any system matrices to be time-varying, taking into account the periodic time-varying nature of the rotorblade system. This LQG control problem, composed of a periodic optimal LQR full-state feedback gain, together with a periodic optimal Kalman-Bucy filter, is amongst the most largely used control design techniques (Athans 1971; Anderson and Moore 1990; Safonov and Athans 1977; Willems and Mitter 1971; Kalman and Bucy 1961). The optimal timevarying regulator and filter gains, which assemble the LQG

\footnotetext{
1 School of Mechanical Engineering University of Campinas

13083-860, Campinas, SP, Brazil

2 Department of Mechanical Engineering

Technical University of Denmark

DK-2800, Kgs. Lyngby, Denmark

Corresponding author:

J. F. Camino, Unicamp, 13083-860, Campinas, SP, Brazil

Email: camino@fem.unicamp.br
} 
controller, are obtained as the solution of some periodic Riccati differential equations (Bittanti et al. 1991b; AbouKandil et al. 2003; Reid 1972; Lancaster and Rodman 1995) whose numerical solutions require the implementation of an appropriate algorithm. Numerical results for rotor-blade systems based on time-varying techniques can be found in Jakobsen et al. (2013); Arcara et al. (2000); Pandiyan and Sinha (1999).

One of the main contributions of this paper, which extends previous results from Jakobsen et al. (2013), is to provide a thorough presentation of the computational application of a periodic time-varying control design strategy capable of attenuating the vibration of coupled rotor-blade systems and of guaranteeing closed-loop stability and performance. The necessary steps to solve the underlying time-varying Riccati equations that arise from the optimal control problem are also presented.

This paper is organized as follows: Section presents the analytic model of the two-dimensional four-blade rotor system under consideration. Section presents the proposed periodic time-varying linear quadratic optimal control designs and the underlying Riccati differential equations. Some preliminary facts regarding periodic systems are provided in Section. Section briefly describes the necessary steps to solve the periodic Riccati differential equations arising in the LQR and the Kalman-Bucy filter problems. In Section, the control strategy is applied to the problem of attenuating the vibrations of the rotorblade system. Numerical simulations show the performance of the proposed design, which is able to cope with the time periodicity of the rotor-blade system and to efficiently suppress tip vibration.

\section{Notation}

This section provides the notation used throughout the paper. The set of complex and real numbers are denoted by $\mathbb{C}$ and $\mathbb{R}$, respectively. The time-derivative of $x(t)$ is denoted by $\dot{x}(t)$. The expected value of a random variable $x$ is denoted by $\mathbb{E}\{x\}$. For a given matrix $M$, its transpose is denoted by $M^{\prime}$ and its inverse by $M^{-1}$. The identity and zero matrices of size $n \times n$ are denoted by $I_{n}$ and $0_{n}$, respectively. The dimension of a matrix is omitted if it is clear from the context. The spectrum of $M$, the set of its eigenvalues, is denoted by $\sigma(M)$. The notation $M \geq 0$ (respectively, $M>0$ ) means that the matrix $M$ is positive semidefinite (respectively, positive definite). The operation $\operatorname{blkdiag}\left(M_{1}, \ldots, M_{n}\right)$ denotes the block diagonal matrix concatenation of its arguments. The image (or range) of a matrix $M$ is denoted by $\operatorname{Im}(M)$. The trace and the determinant of a matrix $M$ is denoted by $\operatorname{tr}(M)$ and $\operatorname{det}(M)$, respectively.

\section{Rotor-blade system}

The mechanical system under investigation is a twodimensional four-blade rotor with tip masses, rotating in a suspended hub, as shown in Figure 1. The hub motion is described by the horizontal position $x_{h}$ and the vertical position $y_{h}$ in the inertial $(x, y)$-coordinate system. For each $i$-th blade, with $i=1, \ldots, 4$, the deflection of an arbitrary point is described by its position $\left(x_{i}, y_{i}\right)$ in the moving local $\left(x_{b_{i}}, y_{b_{i}}\right)$-coordinate system. The angular position of the rotor is given by $\theta(t)$ in the $(x, y)$-coordinate system.

Two actuators are located in the shaft and apply longitudinal forces to the hub on the $(x, y)$-coordinate system, and the other four actuators are located in the blades and apply transverse forces to each blade. Displacement sensors are also located together with the actuators, thus providing the hub position $\left(x_{h}, y_{h}\right)$ and the transverse deflection at the sensor location $\left(x_{i}^{s}, y_{i}^{s}\right)$ on the $i$-th blade, for $i=1, \ldots, 4$. For a detailed explanation, see Christensen (2004); Christensen and Santos (2005).

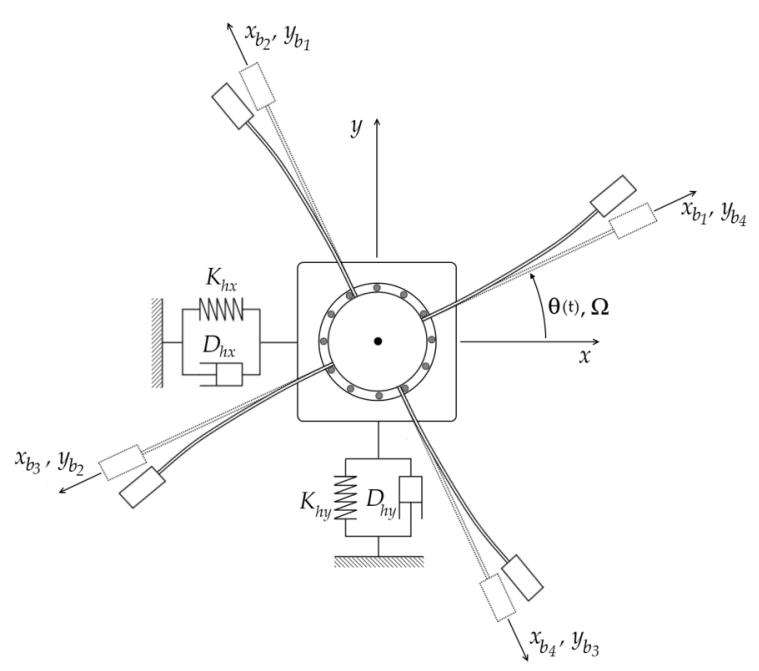

Figure 1. Two-dimensional four-blade rotor system.

The rotor-blade differential equation of motion was derived using the Lagrangian formalism. The resulting model is a time-varying system that depends on the rotor angular position $\theta(t)$ and on the rotational speed $\Omega(t)$. Assuming the system rotates at the constant speed $\Omega(t)=\Omega$, the dynamic model can be rewritten in a periodic linear time-varying form with period $T=2 \pi / \Omega$ given by

$$
M(t) \ddot{z}(t)+D(t) \dot{z}(t)+S(t) z(t)=p(t)+Q_{u} u(t),
$$

where the periodic matrices $M(t)=M(t+T), D(t)=$ $D(t+T)$ and $S(t)=S(t+T)$ represent, respectively, the mass, damping and stiffness of the rotor-blade system, $Q_{u}$ is the control input matrix, $z(t)$ is the generalized coordinate vector, $u(t)$ is the control force that acts on the hub and on the blades, and $p(t)$ represents internal periodic conservative forces originating from the hub unbalance and the unbalance due to the mistuned blades. 
The generalized coordinate vector $z(t)$ is given by

$$
z(t)=\left[\begin{array}{llllll}
x_{h}(t) & y_{h}(t) & q_{1}^{\prime}(t) & q_{2}^{\prime}(t) & q_{3}^{\prime}(t) & q_{4}^{\prime}(t)
\end{array}\right]^{\prime},
$$

where, for each $i$-th blade, the vector $q_{i}(t)$, given by $q_{i}(t)=$ $\left[\begin{array}{lll}q_{i, 1}(t) & \cdots & q_{i, m}(t)\end{array}\right]^{\prime}$, is the generalized coordinate associated with the mode shape $\varphi_{i}(x)$, given by $\varphi_{i}(x)=$ $\left[\begin{array}{lll}\varphi_{i, 1}(x) & \cdots & \varphi_{i, m}(x)\end{array}\right]^{\prime}$. It should be emphasized that the transverse deflection $y_{i}(x, t)$ of the $i$-th blade, at an arbitrary longitudinal position $x$, was derived using the expansion

$$
y_{i}(x, t)=\varphi_{i}^{\prime}(x) q_{i}(t)=\sum_{j=1}^{m} \varphi_{i, j}(x) q_{i, j}(t),
$$

where $m$ is the number of modes. Thus, the total degree of freedom is $N=2+4 \mathrm{~m}$. Notice that the hub degrees of freedom $x_{h}$ e $y_{h}$ are already expressed in physical coordinates, and the $i$-th blade deflection $y_{i}(x, t)$, at an arbitrary position $x$, is a combination of its $m$ modes, as given by (3).

When defining the system output vector $y(t)$ to contain the hub displacements and the transverse deflection at the location of each blade sensor, represented in the system physical coordinate, one has

$$
y(t)=O_{h} z(t)
$$

with $O_{h}$ given by

$$
O_{h}=\left[\begin{array}{cccccc}
1 & 0 & 0 & 0 & 0 & 0 \\
0 & 1 & 0 & 0 & 0 & 0 \\
0 & 0 & \varphi_{1}^{\prime}\left(x_{1}^{s}\right) & 0 & 0 & 0 \\
0 & 0 & 0 & \varphi_{2}^{\prime}\left(x_{2}^{s}\right) & 0 & 0 \\
0 & 0 & 0 & 0 & \varphi_{3}^{\prime}\left(x_{3}^{s}\right) & 0 \\
0 & 0 & 0 & 0 & 0 & \varphi_{4}^{\prime}\left(x_{4}^{s}\right)
\end{array}\right],
$$

where $x_{i}^{s}$ denotes the sensor position on the $i$-th blade. Since the six pairs of sensors and actuators are collocated, it follows that $Q_{u}=O_{h}^{\prime}$.

By defining the state space vector $x(t)=\left[\begin{array}{ll}z^{\prime}(t) & \dot{z}^{\prime}(t)\end{array}\right]^{\prime}$, the equation of motion (1) can be represented in the periodic state-space form

$$
\begin{aligned}
\dot{x}(t) & =A(t) x(t)+B(t) u(t)+f(t) \\
y(t) & =C x(t),
\end{aligned}
$$

with

$$
\begin{aligned}
& A(t)=\left[\begin{array}{cc}
0 & I \\
-M^{-1}(t) S(t) & -M^{-1}(t) D(t)
\end{array}\right], \\
& B(t)=\left[\begin{array}{c}
0 \\
M^{-1}(t) Q_{u}
\end{array}\right], \quad f(t)=\left[\begin{array}{c}
0 \\
M^{-1}(t) p(t)
\end{array}\right] \text {, } \\
& C=\left[\begin{array}{ll}
O_{h} & 0
\end{array}\right]
\end{aligned}
$$

Usually, it is not practical to design a controller to suppress all the modeled modes of the system. In this paper, only the first mode, being the far most significant (Christensen and Santos 2005), is considered for control design. Therefore, the final model will have six degrees of freedom, containing only the two modes related to the hub motion and the first mode of the four blades. All the system matrices used for the numerical simulations (1), taken from Christensen (2004), are included in the appendix.

Remark 1. The control input $u(t)$ can be split into the following two separate contributions: $u(t)=u_{c}(t)+u_{f}(t)$, where $u_{c}(t)$ is the feedback control law, designed using the technique presented in the next sections; and $u_{f}(t), a$ feedforward control law. Since the angular velocity $\Omega$ and the rotor angular position $\theta(t)$ are known, it is possible to cancel out the internal conservative forces $f(t)$, due to unbalances (Jakobsen et al. 2013, and references therein), using the feedforward law

$$
u_{f}(t)=-\left(B^{\prime}(t) B(t)\right)^{-1} B^{\prime}(t) f(t)
$$

Thereby, the external force $f(t)$ is assumed to be zero.

Remark 2. Although it will not be addressed in this paper, the periodic modal decomposition (PMD) used in Christensen and Santos (2005) provides some advantages for control design, since it allows for a straightforward application of modal order reduction and an easier interpretation of the controllability and observability of the rotor-blade system.

\section{Preliminary facts}

This section presents some preliminary facts that play an important role in the theory of linear systems. The source Brockett (1970) is one of the best treatments of the general linear time-varying case. For a thorough discussion on periodic systems, see Yakubovich and Starzhinskii (1975); Bittanti et al. (1991a).

Let $A(t)$ be an $n \times n$ matrix whose elements are bounded continuous functions of time, and consider the time-varying linear system of differential equations

$$
\dot{x}(t)=A(t) x(t)
$$

Then, its fundamental solution is any matrix $X(t)$, whose columns $x^{1}(t), \ldots, x^{n}(t)$ are linearly independent vectors for any $t$ that satisfies

$$
\dot{X}(t)=A(t) X(t),
$$

and its transition matrix $\Phi\left(t, t_{0}\right)$ is the matrix that satisfies

$$
\frac{\mathrm{d}}{\mathrm{d} t} \Phi\left(t, t_{0}\right)=A(t) \Phi\left(t, t_{0}\right), \quad \Phi\left(t_{0}, t_{0}\right)=I
$$

It is well known that the transition matrix can be obtained from a fundamental solution as $\Phi\left(t, t_{0}\right)=X(t) X\left(t_{0}\right)^{-1}$. The transition matrix $\Phi\left(t, t_{0}\right)$ has the following important properties: 
- It satisfies the composition law

$$
\Phi\left(t_{2}, t_{0}\right)=\Phi\left(t_{2}, t_{1}\right) \Phi\left(t_{1}, t_{0}\right), \text { for any } t_{0}, t_{1}, t_{2} .
$$

- It is invertible if $\int_{t_{0}}^{t} \operatorname{tr}(A(\alpha)) \mathrm{d} \alpha$ is finite, as shown by the Abel-Jacobi-Liouville formula

$$
\operatorname{det} \Phi\left(t, t_{0}\right)=\exp \left[\int_{t_{0}}^{t} \operatorname{tr}(A(\alpha)) \mathrm{d} \alpha\right]
$$

The transition matrix characterizes the general solution of the system

$$
\begin{aligned}
& \dot{x}(t)=A(t) x(t)+B(t) u(t) \\
& y(t)=C(t) x(t)+D(t) u(t)
\end{aligned}
$$

as given by the so called variation of constant formula:

$$
x(t)=\Phi\left(t, t_{0}\right) x\left(t_{0}\right)+\int_{t_{0}}^{t} \Phi(t, \tau) B(\tau) u(\tau) \mathrm{d} \tau
$$

When $A(t)$ is $T$-periodic, $A(t+T)=A(t)$, the transition matrix evaluated at $t=T$, denoted by $\Psi_{A}=\Phi(T, 0)$, is called the monodromy matrix of $A(t)$. The matrix $\Psi_{A}$ fully characterizes the stability of the homogeneous periodic systems as shown in the next Lemma 1.

Lemma 1. Yakubovich and Starzhinskii (1975); Brockett (1970). All solutions of the T-periodic equation

$$
\dot{x}(t)=A(t) x(t)
$$

are asymptotically stable if the characteristic multipliers (the Floquet multipliers), which are the roots of the characteristic equation $\operatorname{det}\left(\rho I-\Psi_{A}\right)=0$, lie inside the open unit disk $|\rho|<1$ in the complex plane.

Consider the following $T$-periodic Riccati differential equation

$$
\begin{aligned}
\dot{P}(t)+A^{\prime}(t) P(t) & +P(t) A(t)+Q(t) \\
& -P(t) B(t) R^{-1}(t) B^{\prime}(t) P(t)=0,
\end{aligned}
$$

defined on the interval $-\infty<t<\infty$, subject to the condition $P\left(t_{1}\right)=S \geq 0$, with $S=S^{\prime}, Q(t)=Q^{\prime}(t) \geq 0$, $R(t)=R^{\prime}(t)>0$, and all matrices of compatible size whose elements are bounded continuous functions of time and $T$ periodic. The solution $P(t, S)$ of (17), which passes through $S \neq 0$ at $t=t_{1}$, is $T$-periodic, if $P(t, S)$ is defined and $P(t+T, S)=P(t, S)$ for $-\infty<t<\infty$.

Definitions of stabilizability and controllability (and also detectability and observability) for linear time-varying (LTV) systems are found in Anderson and Moore (1990); Ravi et al. (1990).

Lemma 2. Hewer (1975); Bittanti, Colaneri and Guardabassi (1986). The following statements are equivalent:

1. The pair $(A(t), B(t))$ is stabilizable and the pair $(A(t), D(t))$ is detectable, where $D(t)$ is any matrix such that $D(t) D^{\prime}(t)=Q(t)$.
2. There exists one and only one positive semidefinite $T$ periodic (stabilizing) solution $P(t, S)$ of (17), such that the system

$$
\dot{x}(t)=\left(A(t)-B(t) R^{-1}(t) B^{\prime}(t) P(t, S)\right) x(t)
$$

is asymptotically stable.

Another standard result, which can be found in Brockett (1970), that relates the solution of the (not necessarily periodic) Riccati differential equation with the solution of an associated Hamiltonian system is shown in the next Lemma 3. Comprehensive sources on Riccati equations are Bittanti et al. (1991b); Abou-Kandil et al. (2003); Reid (1972); Lancaster and Rodman (1995).

Lemma 3. Brockett (1970). Let the Hamiltonian matrix $H(t)$ be given by

$$
H(t)=\left[\begin{array}{cc}
A(t) & -B(t) R^{-1}(t) B^{\prime}(t) \\
-Q(t) & -A^{\prime}(t)
\end{array}\right]
$$

For a given symmetric matrix $S$, let $\Phi\left(t, t_{0}\right)$ be the transition matrix of the set of $2 n$ linear differential equations

$$
\left[\begin{array}{c}
\dot{X}(t) \\
\dot{Y}(t)
\end{array}\right]=H(t)\left[\begin{array}{l}
X(t) \\
Y(t)
\end{array}\right], \quad\left[\begin{array}{l}
X\left(t_{1}\right) \\
Y\left(t_{1}\right)
\end{array}\right]=\left[\begin{array}{l}
I \\
S
\end{array}\right]
$$

and let $\Phi\left(t, t_{0}\right)$ be partitioned into $n \times n$ blocks as

$$
\Phi\left(t, t_{0}\right)=\left[\begin{array}{ll}
\Phi_{11} & \Phi_{12} \\
\Phi_{21} & \Phi_{22}
\end{array}\right]
$$

Let $P(t):=P\left(t ; S, t_{1}\right)$ denotes the value at time $t$ of the solution of the Riccati equation (17), which passes through $S$ at $t=t_{1}$. The following statements hold:

1. If $X(t)$ is nonsingular on the interval $\left[t_{0}, t_{1}\right]$, then $P(t)=Y(t) X^{-1}(t)$ is a solution of (17) that satisfies $P\left(t_{1}\right):=P\left(t_{1} ; S, t_{1}\right)=S$.

2. If $P(t):=P\left(t ; S, t_{1}\right)$ is defined as

$$
\begin{aligned}
P(t)= & \left(\Phi_{21}\left(t, t_{1}\right)+\Phi_{22}\left(t, t_{1}\right) S\right) \\
& \times\left(\Phi_{11}\left(t, t_{1}\right)+\Phi_{12}\left(t, t_{1}\right) S\right)^{-1},
\end{aligned}
$$

then $P\left(t_{1}\right):=P\left(t_{1} ; S, t_{1}\right)=S$, and the Riccati equation (17) is satisfied, that is

$$
\begin{aligned}
\dot{P}(t)+ & A^{\prime}(t) P(t)+P(t) A(t) \\
& -P(t) B(t) R^{-1}(t) B^{\prime}(t) P(t)+Q(t)=0
\end{aligned}
$$

The above time-varying matrix $H(t)$ is called Hamiltonian because it satisfies

$$
J H^{\prime}(t) J^{\prime}=-H(t)
$$

for all $t$, with $J$ the skew-symmetric matrix given by

$$
J=\left[\begin{array}{cc}
0 & I \\
-I & 0
\end{array}\right]
$$

It is useful to note that the spectrum of a Hamiltonian matrix is symmetric about the imaginary axis. If $\lambda \in \sigma(M)$, then $-\lambda, \bar{\lambda},-\bar{\lambda} \in \sigma(M)$. 


\section{Algorithm for solving the PRDE}

It is not a trivial task to solve a Riccati differential equation. One might be tempted to directly integrate the equation as an initial value problem, since it is just a system of first order nonlinear differential equations, but this approach usually fails. To see this fact, consider the following scalar Riccati differential equation, obtained from (17), with $P(t)=x(t)$, $A(t)=1, B(t)=2, R(t)=4$, and $Q(t)=3$, given by

$$
\dot{x}=-2 x+x^{2}-3
$$

This differential equation possesses two equilibrium points: $x=-1$, which corresponds to a stable equilibrium, and $x=$ 3 , which corresponds to an unstable equilibrium, as can be easily verified from the phase portrait shown in Figure 2.

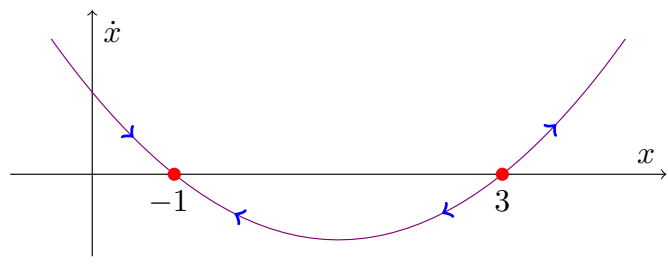

Figure 2. Phase portrait of $\dot{x}=-2 x+x^{2}-3$.

Observe that for any initial condition $x(0)<-1$, the time derivative $\dot{x}$ is positive and, consequently, $x(t)$ will increase until the point $x=-1$ is reached. Likewise, for any initial condition $-1<x(0)<3$, the time derivative $\dot{x}$ is negative and $x(t)$ will decrease until the point $x=-1$ is reached. Thus, the equilibrium $x=-1$ is asymptotically stable. On the other hand, starting from any initial condition $x(0)>3$, the solution of (26) diverges, since $\dot{x}$ is positive in this region. Note, however, that a backward integration from any initial condition $x(0)>-1$ will eventually converge to the equilibrium $x=3$.

Actually, since the Riccati differential equation is a nonlinear equation, it is not clear that a solution will exist for a given initial condition. The well-known Picard-Lindelöf theorem on differential equations (Hartman 2002; Hale 1969) guarantees (under the hypothesis that the functions are locally Lipschitz) only local existence and uniqueness for the solution of a nonlinear equation; without further analysis, it is not possible to conclude existence over longer intervals because of the phenomenon of finite escape time. For instance, consider the scalar equation $\dot{x}=1+x^{2}$, with an initial condition $x(0)=0$, whose solution is $x(t)=$ $\tan (t)$. Clearly, this solution escapes to infinity as $t \rightarrow \pi / 2$.

As pointed out in the groundbreaking paper by Kalman (1960), one of the preliminary works to provide conditions for the existence of a solution for the Riccati differential equation (17) and the underlying optimal linear quadratic problem, one cannot, in general, compute, for the timevarying case, a solution $P(t)$ as $t \rightarrow \infty$ from the knowledge of an initial condition $S$. However, when all the coefficients of the Riccati equation are constant, one can compute the solution by integrating in reverse time from any initial condition $S \geq 0$. Further contributions can be found in Wonham (1968); Hewer (1975); Bekir and Bucy (1976) and references therein. If the coefficients of the Riccati equation are $T$-periodic (Bittanti et al. 1991a), then the solution $P(t)$ must also be periodic, i.e., the boundary condition $P(t+$ $T)-P(t)=0$ needs to be satisfied. Thus, a suitable initial condition is necessary, which can be provided by the so called periodic generator at $t_{o}$, i.e., the value $P\left(t_{o}\right)$ taken by a periodic solution $P(\cdot)$ at time $t_{o}$.

Before presenting an algorithm to solve the periodic Riccati differential equation, it is appropriate to discuss the linear time-invariant case. For this case, Kučera (1972) provided necessary and sufficient conditions, under which the algebraic Riccati equation

$$
A^{\prime} P+P A-P B R^{-1} B^{\prime} P+Q=0,
$$

with $R=R^{\prime}>0$ and $Q=Q^{\prime} \geq 0$, has a unique stabilizing solution $P=P^{\prime} \geq 0$. A popular approach to compute this solution, which originates with Anderson (1966) and Potter (1966), is based on invariant subspace methods, in which the solution $P$ of (27) is characterized in terms of the eigensystem of the associated Hamiltonian matrix

$$
H=\left[\begin{array}{cc}
A & -B R^{-1} B \\
-Q & -A^{\prime}
\end{array}\right],
$$

as shown in Lemma 4 and Lemma 5, which bear some connections with Lemma 3.

Lemma 4. Let $\mathcal{V} \in \mathbb{C}^{2 n \times n}$ be an n-dimensional invariant subspace of $H$, and let $Y, X \in \mathbb{C}^{n \times n}$ be two complex matrices such that

$$
\mathcal{V}=\operatorname{Im}\left[\begin{array}{l}
X \\
Y
\end{array}\right]
$$

If $X$ is invertible, then $P=Y X^{-1}$ is a solution to the algebraic Riccati equation (27), and $\sigma\left(A-B R^{-1} B X\right)=$ $\sigma\left(\left.H\right|_{\mathcal{V}}\right)$, where $\left.H\right|_{\mathcal{V}}$ is the restriction of $H$ to $\mathcal{V}$. Furthermore, the solution $P$ is independent of a specific choice of bases of $\mathcal{V}$.

Lemma 5. If $P \in \mathbb{C}^{n \times n}$ is a solution to the Riccati equation (27), then there exist matrices $Y, X \in \mathbb{C}^{n \times n}$, with $X$ invertible, such that $P=Y X^{-1}$ and the columns of $\left[\begin{array}{l}X \\ Y\end{array}\right]$ form a basis of an $n$-dimensional invariant subspace of $H$.

From the above lemmas, it is clear that, to compute a solution to the algebraic Riccati equation (27), it is necessary to construct bases for the invariant subspace of $H$, which is accomplished via an appropriate eigensystem decomposition. As shown in Laub (1979, 1991), most reliable algorithms to compute numerical solutions of the Algebraic Riccati equation are based on invariant subspace methods, implemented using Schur decomposition 
techniques. See also Kenney and Lepnik (1985); Lancaster and Rodman (1995) for an overview of numerical methods to solve algebraic Riccati equations.

The extension of this eigensystem decomposition to the periodic time-varying case is given in Kano and Nishimura (1979), which shows how to compute the $T$ periodic positive semidefinite stabilizing (maximal) solution of the periodic Riccati differential equation (17). The solution is computed from the monodromy matrix of the associated Hamiltonian system, by applying an appropriate ordering of the invariant subspace into its stable and antistable parts. To improve numerical efficacy, Hench et al. (1994) presented a symplectic integration algorithm that preserves the integral invariants of Hamiltonian systems. The algorithm described in Varga (2008) to compute a $T$-periodic positive semidefinite matrix solution $P(t)$ of the periodic Riccati differential equation (17) is now presented.

\section{ALGORITHM:}

1. Construct the $T$-periodic Hamiltonian matrix

$$
H(t)=\left[\begin{array}{cc}
A(t) & -B(t) R^{-1}(t) B^{\prime}(t) \\
-Q(t) & -A^{\prime}(t)
\end{array}\right]
$$

2. Compute the monodromy matrix $\Psi_{H}$ by integrating the Hamiltonian system from 0 to $T$

$$
\dot{X}(t)=H(t) X(t), \quad X(0)=I
$$

The monodromy matrix $\Psi_{H}$ is thus given by $\Psi_{H}=$ $X(T)$. It is a symplectic matrix, i.e., $\Psi_{H}$ satisfies the condition

$$
\Psi_{H}^{\prime} J \Psi_{H}=J
$$

with the skew-symmetric matrix $J$ given by (25). Notice that symplectic matrices have the important property that the eigenvalues occur in reciprocal pairs, that is, if $\lambda \neq 0$ is an eigenvalue, then so is $\lambda^{-1}$.

3. Apply the ordered Schur decomposition $\Psi_{H}=$ $U Z U^{\prime}$, with $U^{\prime} U=I$ as follows:

$$
\begin{array}{r}
{\left[\begin{array}{ll}
U_{11} & U_{12} \\
U_{21} & U_{22}
\end{array}\right]^{\prime}\left[\begin{array}{ll}
\Psi_{11} & \Psi_{12} \\
\Psi_{21} & \Psi_{22}
\end{array}\right]\left[\begin{array}{ll}
U_{11} & U_{12} \\
U_{21} & U_{22}
\end{array}\right]=} \\
{\left[\begin{array}{cc}
Z_{11} & Z_{12} \\
0 & Z_{22}
\end{array}\right]}
\end{array}
$$

where $Z_{11} \in \mathbb{R}^{n \times n}$ is upper quasi-triangular with $n$ eigenvalues inside the open unit circle, and $Z_{22} \in$ $\mathbb{R}^{n \times n}$ is upper quasi-triangular with $n$ eigenvalues outside the unit circle.

4. Integrate from 0 to $T$ the Hamiltonian system

$$
\dot{Y}(t)=H(t) Y(t), \quad Y(0)=\left[\begin{array}{c}
U_{11} \\
U_{21}
\end{array}\right]
$$

5. Finally, compute the solution of the periodic Riccati equation as

$$
P(t)=Y_{2}(t) Y_{1}^{-1}(t)
$$

with $Y_{1}(t)$ and $Y_{2}(t)$ the partitions of matrix $Y(t)$, given by $Y(t)=\left[\begin{array}{l}Y_{1}(t) \\ Y_{2}(t)\end{array}\right]$

\section{Control design}

The linear quadratic regulator (LQR) problem is one of the most largely used methodologies to design full-state feedback controllers (Athans 1971; Anderson and Moore 1990), which have impressive robust stability properties for linear time-invariant (LTI) systems that include at least $\pm 60^{\circ}$ phase margin, infinite gain margin, and 50 percent gainreduction tolerance (Safonov and Athans 1977).

For the infinite-horizon continuous-time LTI case, the optimal full-state feedback gain is obtained as the solution of an algebraic Riccati equation. On the other hand, for the finite-horizon LTI case, or if any of the system matrices are time-varying, the optimal full-state feedback gain is obtained as the solution of a Riccati differential equation. Since the formulation presented in this paper holds for any linear timevarying system, the formulas also hold for periodic systems, as a particular case.

\section{Optimal LQG control problem}

The central element of the LQR problem is the design of a static gain $G(t)$ for the full-state feedback law

$$
u(t)=-G(t) x(t)
$$

for the linear time-varying system

$$
\dot{x}(t)=A(t) x(t)+B(t) u(t), \quad x(0)=x_{0}
$$

that minimizes the quadratic cost function

$$
\mathcal{J}_{\mathrm{LQR}}=\int_{0}^{\infty}\left(x^{\prime}(t) Q(t) x(t)+u^{\prime}(t) R(t) u(t)\right) \mathrm{d} t
$$

in which the time-varying matrices $Q(t)=Q^{\prime}(t) \geq 0$ and $R(t)=R^{\prime}(t)>0$ penalize, respectively, the system states $x(t)$ and the control input $u(t)$. The next Lemma 6 characterizes the solution of the LQR problem.

Lemma 6. Anderson and Moore (1990). Assume the matrices $A(t), B(t), Q(t)=Q^{\prime}(t) \geq 0$ and $R(t)=R^{\prime}(t)>$ 0 are bounded continuous functions of time. Furthermore, assume the pair $(A(t), B(t))$ is completely controllable for every time $t$. Then, the optimal time-varying full-state feedback law is $u(t)=-G(t) x(t)$ with the gain given by

$$
G(t)=R^{-1}(t) B^{\prime}(t) P(t)
$$

where the positive semidefinite matrix $P(t)=P^{\prime}(t)$ satisfies the Riccati differential equation

$$
\begin{aligned}
\dot{P}(t)+A^{\prime}(t) P(t)+ & P(t) A(t) P(t)+Q(t) \\
& -P(t) B(t) R^{-1}(t) B^{\prime}(t)=0
\end{aligned}
$$


Moreover, the gain $G(t)$ guarantees asymptotic stability of the closed-loop system

$$
\dot{x}(t)=\left(A(t)-B(t) R^{-1}(t) B^{\prime}(t) P(t)\right) x(t),
$$

and the minimum value of the performance index (38) is $\mathcal{J}_{L Q R}^{*}=x_{0}^{\prime} P(0) x_{0}$.

The LQR control law (36) requires a direct measurement of all system states, which in many practical applications is either impossible or impractical. However, one can resort to an observer to estimate the states. This is the cornerstone of the linear quadratic Gaussian (LQG) problem (Athans 1971; Bittanti et al. 1990; Green and Limebeer 1995; Casti 1980), which minimizes a quadratic integral performance index subject to linear state-space dynamics driven by Gaussian white noise. The LQG design, for which the separation principle (Willems and Mitter 1971) plays an important role, is a combination of an optimal (full-state) observer with an optimal (full-state) feedback gain.

The optimal Kalman-Bucy filter for system (37) has the structure of the Luenberger observer

$$
\dot{\hat{x}}(t)=A(t) \hat{x}(t)+B(t) u(t)+L(t)(y(t)-C(t) \hat{x}(t))
$$

where all the matrices are assumed to have compatible size, $\hat{x}(t)$ is the state estimate provided by the filter, $L(t)$ is the filter gain, $\hat{x}(0)=\mathbb{E}\left\{x_{0}\right\}$ is the initial state estimate, and the system model (37) is now assumed to be corrupted by noise as follows:

$$
\begin{aligned}
& \dot{x}(t)=A(t) x(t)+B(t) u(t)+w(t) \\
& y(t)=C(t) x(t)+v(t),
\end{aligned}
$$

with $w(t)$ and $v(t)$ being, respectively, process and measurement noises. Moreover, it is assumed that $w(t)$ and $v(t)$ are uncorrelated zero-mean Gaussian white noise, with covariance matrices respectively given by

$$
\mathbb{E}\left\{\left[\begin{array}{l}
w(t) \\
v(t)
\end{array}\right]\left[\begin{array}{ll}
w^{\prime}(\tau) & v^{\prime}(\tau)
\end{array}\right]\right\}=\left[\begin{array}{cc}
W(t) & 0 \\
0 & V(t)
\end{array}\right] \delta(t-\tau),
$$

where $\delta(t)$ is the Diract delta function. Thus, the matrices $W(t)=W^{\prime}(t) \geq 0$ and $V(t)=V^{\prime}(t)>0$ are used to model process and observation noise, respectively. The next Lemma 7 characterizes the solution of the optimal estimation problem.

Lemma 7. Kalman and Bucy (1961). The optimal KalmanBucy filter gain $L(t)$ that minimizes $\mathbb{E}\left\{e^{\prime}(t) e(t)\right\}$, with $e(t)=x(t)-\hat{x}(t)$ the estimation error, is given by

$$
L(t)=Y(t) C^{\prime}(t) V^{-1}(t),
$$

in which the estimation error covariance matrix $Y(t)=$ $Y^{\prime}(t)>0$ is the solution of the Riccati differential equation $(R D E)$

$$
\begin{aligned}
& -\dot{Y}(t)+A(t) Y(t)+Y(t) A^{\prime}(t)+W(t) \\
& -Y(t) C^{\prime}(t) V^{-1}(t) C(t) Y(t)=0
\end{aligned}
$$

Since the separation principle holds, the design of both gains can be performed independently. Thus, the control law using the observer is given by

$$
u(t)=-G(t) \hat{x}(t),
$$

in which the optimal gain $G(t)$, given by (39), is the solution of the LQR problem, and the optimal estimate $\hat{x}(t)$ is given by the Kalman-Bucy filter (42). Figure 3 shows the structure of the separation principle for the LQG problem.

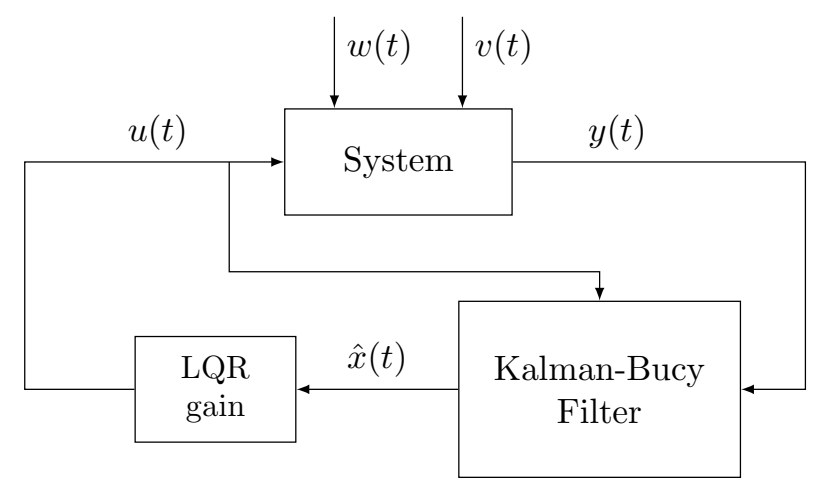

Figure 3. The separation principle.

The LQG controller is, thus, a dynamic, strictly proper controller that has the following state-space realization:

$$
\begin{aligned}
\dot{x}_{c}(t) & =A_{c}(t) x_{c}(t)+B_{c}(t) y(t) \\
u(t) & =C_{c}(t) x_{c}(t),
\end{aligned}
$$

with the matrices $A_{c}(t), B_{c}(t)$ and $C_{c}(t)$ given by

$$
\begin{aligned}
& A_{c}(t)=A(t)-B(t) G(t)-L(t) C(t), \\
& B_{c}(t)=L(t) \\
& C_{c}(t)=-G(t)
\end{aligned}
$$

Using the fact that $G(t)=R^{-1}(t) B^{\prime}(t) P(t)$ and $L(t)=$ $Y(t) C^{\prime}(t) V^{-1}(t)$, the system matrices become

$$
\begin{aligned}
A_{c}(t)=A(t)-B(t) R^{-1}(t) B^{\prime}(t) P(t) & \\
& \quad-Y(t) C^{\prime}(t) V^{-1}(t) C(t) \\
B_{c}(t)=Y(t) C^{\prime}(t) V^{-1}(t), & \\
C_{c}(t)= & -R^{-1}(t) B^{\prime}(t) P(t),
\end{aligned}
$$

with $P(t)$ and $Y(t)$ the solution of the following Riccati differential equations:

$$
\begin{aligned}
\dot{P}(t)+A^{\prime}(t) P(t) & +P(t) A(t)+Q(t) \\
& -P(t) B(t) R^{-1}(t) B^{\prime}(t) P(t)=0
\end{aligned}
$$

and

$$
\begin{aligned}
& -\dot{Y}(t)+A(t) Y(t)+Y(t) A^{\prime}(t)+W(t) \\
& -Y(t) C^{\prime}(t) V^{-1}(t) C(t) Y(t)=0
\end{aligned}
$$

The LQG controller is optimal in the sense that it minimizes the quadratic performance index

$\mathcal{J}_{\text {LQG }}=\lim _{T \rightarrow \infty} \mathbb{E}\left\{\frac{1}{T} \int_{0}^{T}\left(x^{\prime}(t) Q(t) x(t)+u^{\prime}(t) R(t) u(t)\right) \mathrm{d} t\right\}_{(53)}$ 
The closed-loop augmented system, composed of the plant states $x(t)$ and the observer error $e(t)=x(t)-\hat{x}(t)$, is given by

$$
\begin{array}{r}
{\left[\begin{array}{c}
\dot{x}(t) \\
\dot{e}(t)
\end{array}\right]=\left[\begin{array}{cc}
A(t)-B(t) G(t) & B(t) G(t) \\
0 & A(t)-L(t) C(t)
\end{array}\right]\left[\begin{array}{l}
x(t) \\
e(t)
\end{array}\right]} \\
+\left[\begin{array}{cc}
I & 0 \\
I & -L(t)
\end{array}\right]\left[\begin{array}{l}
w(t) \\
v(t)
\end{array}\right]
\end{array}
$$

with $G(t)$ the LQR gain and $L(t)$ the Kalman-Bucy filter gain. From the block diagonal structure, it is clear that stability properties of the augmented closed-loop system are characterized by the stability properties of the blocks $A(t)-B(t) G(t)$ and $A(t)-L(t) C(t)$. Thus, the gains $G(t)$ and $L(t)$ can be independently designed, such that the systems $\dot{x}(t)=(A(t)-B(t) G(t)) x(t)$ and $\dot{e}(t)=$ $(A(t)-L(t) C(t)) e(t)$ are asymptotically stable.

\section{Numerical results}

This section analyses the performance of the rotor-blade system in closed-loop with the time-varying linear quadratic Gaussian (LQG) controller designed using the approach described in Section .

The system under consideration is described by the state-space model (6) in Section . Notice that $f(t)=0$, since the unbalance is neglected as pointed in Remark 1. For this system, the initial condition has been chosen as $z(0)=\left[\begin{array}{llllll}1 & 1 & 1 & 1 & -1 & -1\end{array}\right][\mathrm{mm}]$ and $\dot{z}(0)=0$ $[\mathrm{mm} / \mathrm{s}]$, meaning that the hub is initially deflected $1[\mathrm{~mm}]$ in both the $(x, y)$-direction, and the first mode of each one of the blades are deflected $1[\mathrm{~mm}]$. The reason for the negative sign of the third and fourth blades is to make them move in phase with the opposite blades, instead of completely out of phase, which would effectively cancel out the bladehub coupling (Jakobsen et al. 2013; Christensen and Santos 2005). The angular velocity is set at $\Omega=300$ [rpm].

First, the stability of the open-loop system $\dot{x}(t)=$ $A(t) x(t)$ is analyzed using Lemma 1. Computing the monodromy matrix $\Psi_{A}$ associated with $A(t)$, the absolute value of the Floquet multipliers are found to be 0.9007 , $0.9007,0.8939,0.8939,0.7262,0.7262,0.6084,0.6084$, $0.6084,0.6084,0.6015,0.6015$, which lie inside the open unit circle in the complex plane. Thus, the equilibrium at the origin of the open-loop system is already asymptotically stable. Since the largest absolute value 0.9007 (the least stable Floquet multiplier) is located relatively close to the unit circle, one can expect a weakly damped behavior.

Remark 3. All numerical integrations are performed using the Matlab ode suit, with stringent error tolerances. Although it was not necessary, an integrator with structurepreserving (symplectic) techniques (Hairer et al. 2006) might provide more accurate solutions.
Assuming that all states are available for feedback, an LQR controller is designed. The key aspect of this design relies on the choice of the weighting matrices $Q(t)=$ $Q^{\prime}(t) \geq 0$ and $R(t)=R^{\prime}(t)>0$ that penalize, respectively, the system state $x(t)$ and the control input $u(t)$. Most weighting selection methods found in the literature are performed through an intuitive trial-and-error approach, with the matrices chosen as diagonal. For the LTI case, a procedure for selecting the weighting matrices can be found in Harvey and Stein (1978). However, a selection procedure for the LTV case is indubitably far from trivial. Since the goal is to suppress blade tip vibration, only the states related to tip deflection (and its time-derivative) are weighted. Thus, matrix $Q(t)$ is chosen as a constant matrix given by

$$
Q=10^{2} \times \operatorname{blkdiag}\left(0_{2}, I_{4}, 0_{2}, I_{4}\right)
$$

The weighting matrix $R(t)$ is also taken to be constant and given by $R=10^{-1} I_{6}$, which equally penalizes all control inputs. This selection seems to be a reasonable choice.

Following the algorithm presented in Section, the $T$ periodic Hamiltonian matrix $H(t)$, associated with the Riccati equation (51), is constructed and its monodromy matrix $\Psi_{H}$ is computed. As stated in Section, the monodromy matrix must be symplectic, which is readily verified since $\left\|\Psi_{H}^{\prime} J \Psi_{H}-J\right\|_{2}=6.3173 \times 10^{-7}$.

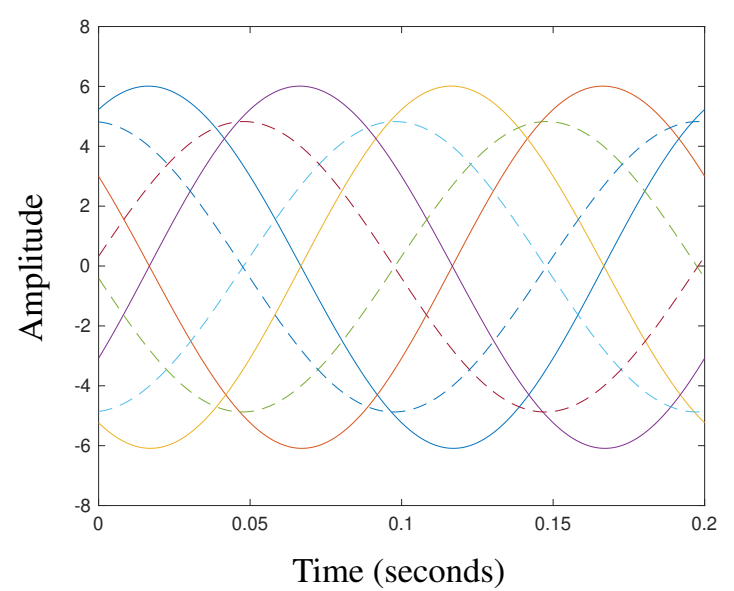

Figure 4. Some of the time-varying elements of matrix $P(t)$ : the entries $P_{1, j} \times 10^{-3}$ for $j=3, \ldots, 6$ (in solid line) and $P_{1, j} \times 10^{-1}$ for $j=9, \ldots, 12$ (in dashed line).

After applying the Schur decomposition described in step 3 of the Algorithm, the solution $P(t)$ is computed. Since $P(t)$ is a symmetric matrix of size $12 \times 12$, which might have up to 78 distinct (time-varying) elements, it is impractical to plot all of them. Figure 4 shows some of the time-varying elements of $P(t)$ : the entries $P_{1, j} \times 10^{-3}$ for $j=3, \ldots, 6$ (in solid line) and $P_{1, j} \times 10^{-1}$ for $j=$ $9, \ldots, 12$ (in dashed line). The scaling was added so that all data can fit in the same plot. Most of the other time-varying elements follow a similar pattern. The matrix $P(t)$ also has some constant elements. 


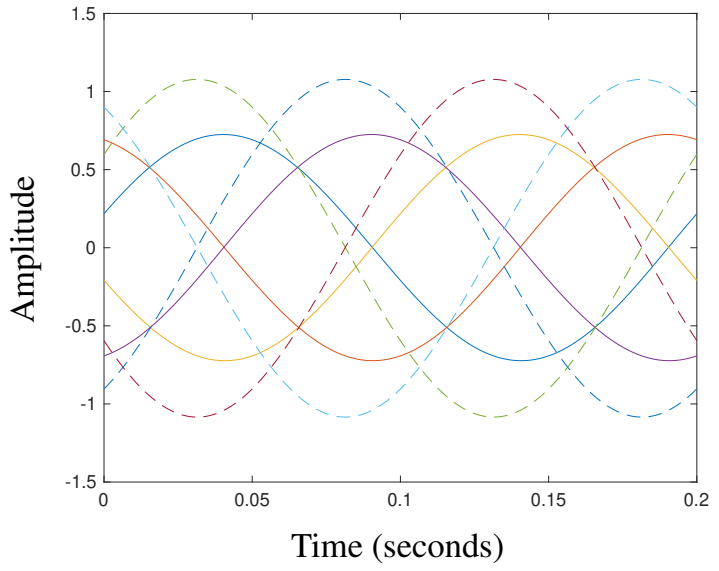

Figure 5. Some of the time-varying elements of matrix $G(t)$ : the entries $G_{1, j} \times 10^{-2}$ for $j=3, \ldots, 6$ (in solid line) and $G_{1, j}$ for $j=9, \ldots, 12$ (in dashed line).

As discussed in the previous sections, the stabilizing solution $P(t)$ of the Riccati equation (51) must be positive semidefinite. This is the case, since the eigenvalues of the matrix $P(t)$, evaluated at a fine time grid, are constant and given by $1.4466,1.4689,1.5648,1.5649,30.273,37.611$, 19358, 19407, 20045, 20066, $2.0199 \times 10^{5}, 2.9956 \times 10^{5}$.

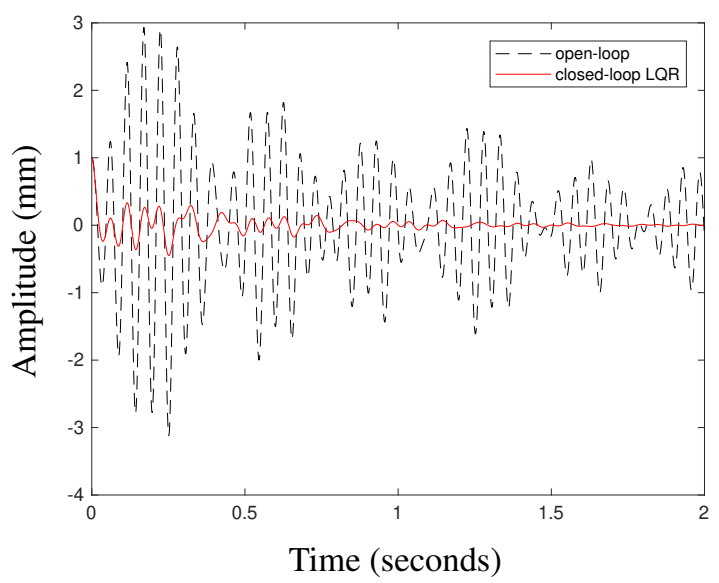

Figure 6. Blade 1 tip position.

Recall that the $T$-periodic time-varying LQR gain is given by $G(t)=-R^{-1}(t) B^{\prime}(t) P(t)$. Figure 5 shows some of the elements of $G(t)$ : the entries $G_{1, j} \times 10^{-2}$ for $j=$ $3, \ldots, 6$ (in solid line) and $G_{1, j}$ for $j=9, \ldots, 12$ (in dashed line). With this gain, the closed-loop system is given by $\dot{x}(t)=(A(t)-B(t) G(t)) x(t)$. The absolute value of the monodromy matrix Floquet multipliers associated with this closed-loop system are: $0.7208,0.7208,0.6037,0.6037$, $0.0024,0.0024,0.0031,0.0031,0.0028,0.0028,0.0028$, 0.0028 . Note that the smallest multiplier moved from 0.6015 in open-loop to 0.0028 in closed-loop. Moreover, the least stable Floquet multiplier moved from 0.9007 in open-loop to 0.7208 in closed-loop, providing, thus, significantly higher stability and robustness margins.

Figure 6 and Figure 7 show, respectively, blade 1 tip position and hub position in the $x$-direction, for the openloop and the closed-loop systems with the LQR controller

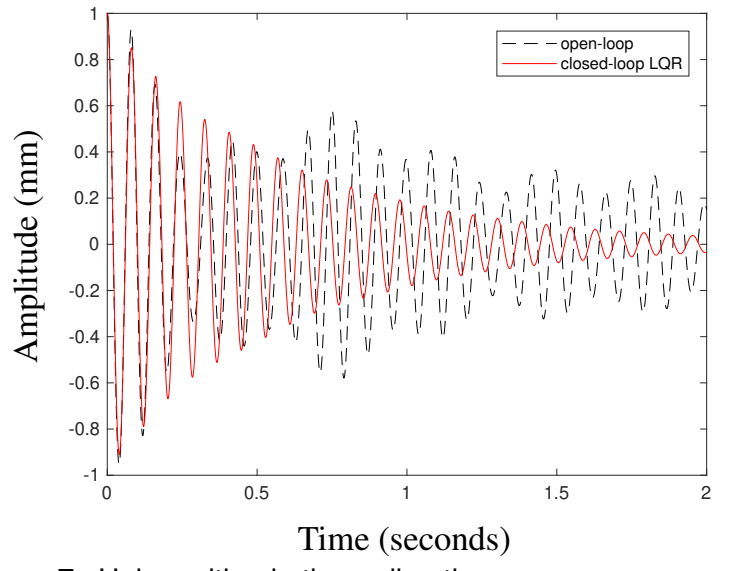

Figure 7. Hub position in the $x$-direction.

$G(t)$. Blades 2, 3 and 4 have a qualitatively similar behavior as blade 1. Likewise, the behavior of the hub position in the $y$-direction is also similar to its behavior in the $x$-direction. Figure 6 shows that the vibration of the blade tip deflection was significantly damped and reduced with a fast settling time. On the other hand, the vibration of the hub position in closed-loop also decreased, but was not heavily damped as the behavior of the blade 1 tip position. This behavior was expected, since the choice of the weighting matrix $Q$ only penalized blade deflection.

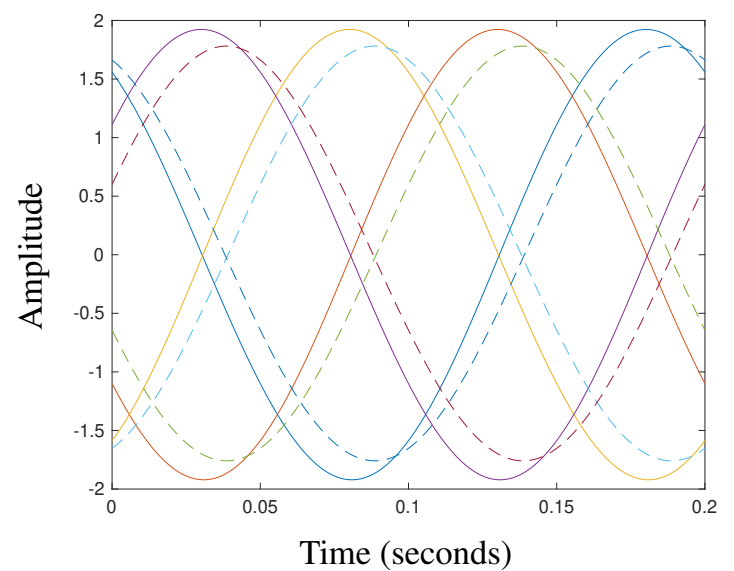

Figure 8. Some of the time-varying elements of matrix $Y(t)$ : the entries $Y_{1, j}$ for $j=3, \ldots, 6$ (in solid line) and $Y_{1, j} \times 10^{-2}$ for $j=9, \ldots, 12$ (in dashed line).

Assuming now that not all states are available for feedback, let us design the observer given by (42), using as measurements the system output vector $y(t)$ given by (4). The weighting matrices $W$ and $V$ for the Kalman-Bucy filter are taken to be constant, given by $W=10^{-3} I$ and $V=10^{-4} I$. Following the steps of the algorithm in Section, the monodromy matrix of the $T$-periodic Hamiltonian matrix $H(t)$, associated with the Riccati equation (52) and its solution $Y(t)$, were computed. Figure 8 shows some of the time-varying elements of matrix $Y(t)$ : the entries $Y_{1, j}$ for $j=3, \ldots, 6$ (in solid line) and $Y_{1, j} \times 10^{-2}$ for $j=$ $9, \ldots, 12$ (in dashed line). 


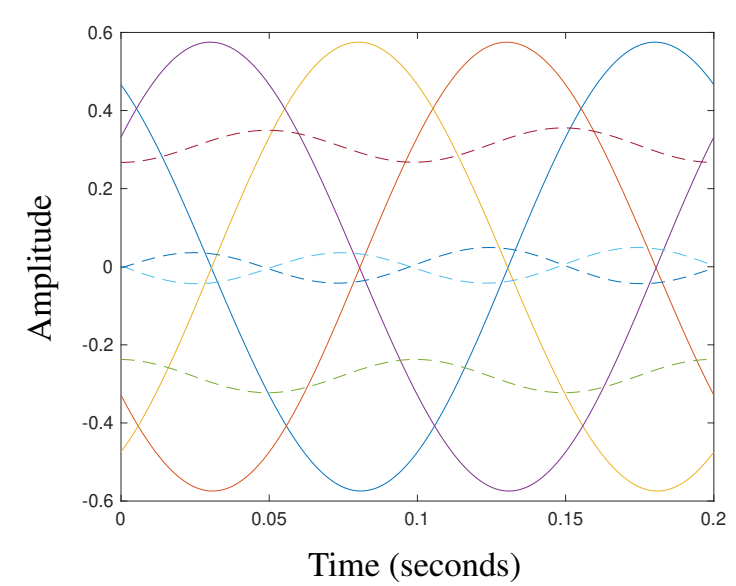

Figure 9. Some of the time-varying elements of matrix $L(t)$ : the entries $L_{1, j}$ for $j=3, \ldots, 6$ (in solid line) and $L_{5, j} \times 10^{-1}$ for $j=3, \ldots, 6$ (in dashed line).

The eigenvalues of $Y(t)$, evaluated at a fine time grid, are constant and given by $0.70231,0.99745,0.99799,1.1814$, $3.3712,4.7654,6027.1,12771,12784,139932.3864 \times$ $10^{5}, 2.7807 \times 10^{5}$. So, matrix $Y(t)$ is positive semidefinite as expected. With this matrix solution $Y(t)$, the KalmanBucy filter gain $L(t)$ is given by $L(t)=Y(t) C^{\prime}(t) V^{-1}(t)$. Figure 9 shows some of the time-varying elements of $L(t)$ : the entries $L_{1, j}$ for $j=3, \ldots, 6$ (in solid line) and $L_{5, j} \times$ $10^{-1}$ for $j=3, \ldots, 6$ (in dashed line).

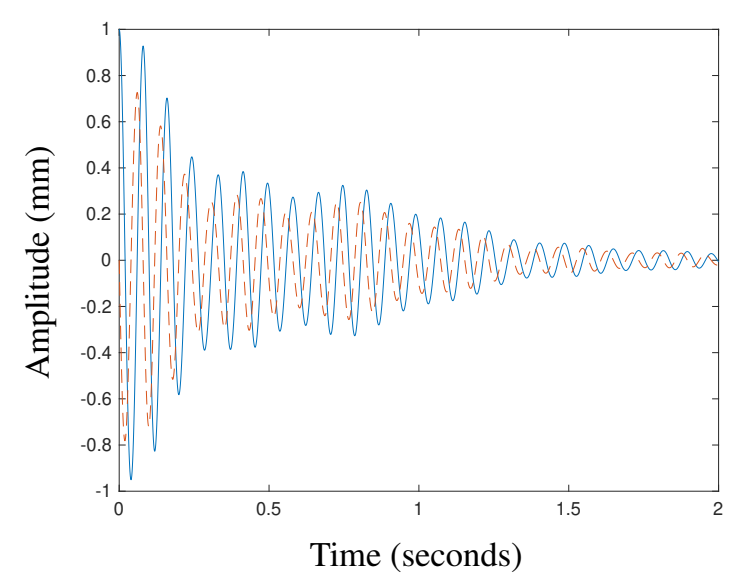

Figure 10. Entries 1 (in solid line) and 7 (in dashed line) of the estimation error $e(t)=x(t)-\hat{x}(t)$.

The Floquet multipliers of the filter closed-loop matrix $A(t)-L(t) C(t)$ are: $0.68831,0.68831,0.68608,0.68608$, 0.63198, 0.63198, 0.60297, 0.60297, 0.60297, 0.60297, $0.59315,0.59315$, which lie inside the open unit circle in the complex plane. Thus, the observer is asymptotically stable. Since the least stable Floquet multiplier is 0.68831 , one can expect some oscillations in estimation error. Figure 10 shows the entries 1 (in solid line) and 7 (in dashed line) of the estimation error $e(t)=x(t)-\hat{x}(t)$, where the estimator initial condition is taken as zero. Clearly, the estimation error $e(t)$ asymptotically converges to zero.

From the separation principle, the augmented closedloop system with the dynamic LQG controller (48) is also

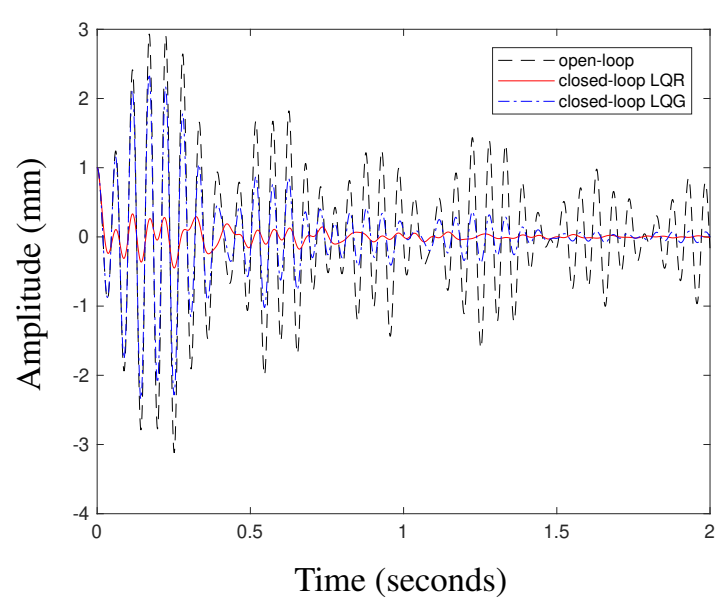

Figure 11. Blade 1 tip deflection for the system in: open-loop (dashed-line), closed-loop using LQR controller (solid line), closed-loop using LQG controller (dash-dot line).

asymptotically stable. Figure 11 shows the blade 1 tip deflection of the open-loop system (in dashed line), the closed-loop system with the LQR controller (in solid line) and the closed-loop system with the LQG controller (in dashed-dot line). There is some decrease in performance by using the LQG controller instead of the LQR controller, but the LQG controller still provides significant damping compared to the open-loop system.

\section{Conclusion}

Attenuation of the tip vibration of a rotor-blade mechanical system is a challenging problem due to the periodic timevarying nature of the system dynamics. To tackle this problem, periodic time-varying LQR and LQG controllers were designed based on a linear time-varying model of the system. The steps to solve the underlying periodic timevarying Riccati differential equation, which is not a trivial task, were presented in details. It should be emphasized that both optimal time-varying LQR and LQG designs guarantee closed-loop stability and performance for the time-varying equation of motion.

The linear time-varying (LTV) periodic model, borrowed from Christensen (2004); Christensen and Santos (2005), describes the dynamics of the rotor-blade system with six degrees of freedom: two related to hub motion and one for each blade transverse deflection. The system is fully actuated and the rotor is assumed to rotate at constant speed.

For this model, a periodic LQR controller was first designed. The goal of the controller is to attenuate blade tip deflection. Thus, the weighting used in the LQR performance index penalized only the control input and the states related to tip deflection, neglecting the states related to hub motion. These matrices were chosen as constant and diagonal. There exists no clear approach to the selection of weighting matrices for time-varying linear-quadratic optimal control design. One can always resort to trial and error. The periodic LQR controller improved system performance significantly 
by highly damping the modes related to blade tip deflection. Thus, tip vibration strongly decreased compared to the openloop case.

A periodic time-varying observer, based on the optimal Kalman-Bucy filter, was proposed in case not all states are available for feedback. Again, the weighting matrices for the quadratic performance index were chosen to be constant and diagonal. The measured output used for the observer was obtained from the six sensors, placed at the same location as the actuators. The estimation error, between the system states and the observer states, asymptotically converged to zero, but not abruptly.

The periodic LQG controller, comprised by the timevarying optimal LQR and Kalman-Bucy filter gains, provided inferior performance compared to the periodic LQR controller. This is expected, since the system states are replaced by their estimation. However, the LQG controller still improved the closed-loop system performance compared to the open-loop case. Eventually, different weighting selection for the LQR and the Kalman-Bucy filter would lead to different performance. Both designs ensure stability. This fact was verified by computing the Floquet multipliers of the closed-loop systems.

\section{Acknowledgments}

The authors gratefully acknowledge the financial support from Fundação de Amparo à Pesquisa do Estado de São Paulo (FAPESP), grant 2018/24867-7.

\section{Appendix}

This section presents the data used for the numerical simulations. The system matrices for $\Omega=300 \mathrm{rpm}$ and period $T=2 \pi / \Omega=0.2$ seconds are given by

$$
M(t)=\left[\begin{array}{ccc}
10.99 & 0 & -0.135 S_{0} \\
0 & 9.09 & 0.135 C_{0} \\
-0.135 S_{0} & 0.135 C_{0} & 0.161 \\
-0.135 S_{1} & 0.135 C_{1} & 0 \\
-0.135 S_{2} & 0.135 C_{2} & 0 \\
-0.135 S_{3} & 0.135 C_{3} & 0 \\
-0.135 S_{1} & -0.135 S_{2} & -0.135 S_{3} \\
0.135 C_{1} & 0.135 C_{2} & 0.135 C_{3} \\
0 & 0 & 0 \\
0.161 & 0 & 0 \\
0 & 0.161 & 0 \\
0 & 0 & 0.161
\end{array}\right]
$$

$$
D(t)=\left[\begin{array}{ccc}
1.200 & 0 & -0.270 \Omega C_{0} \\
0 & 1.500 & -0.270 \Omega S_{0} \\
0 & 0 & 0.800 \\
0 & 0 & 0 \\
0 & 0 & 0 \\
0 & 0 & 0
\end{array}\right.
$$$$
\left.\begin{array}{ccc}
-0.270 \Omega C_{1} & -0.270 \Omega C_{2} & -0.270 \Omega C_{3} \\
-0.270 \Omega S_{1} & -0.270 \Omega S_{2} & -0.270 \Omega S_{3} \\
0 & 0 & 0 \\
0.800 & 0 & 0 \\
0 & 0.800 & 0 \\
0 & 0 & 0.800
\end{array}\right]
$$

$S(t)=\left[\begin{array}{ccc}6.6 \times 10^{4} & 0 & 0.135 \Omega^{2} S_{0} \\ 0 & 7.7 \times 10^{4} & -0.135 \Omega^{2} C_{0} \\ 0 & 0 & \hat{S}_{0} \\ 0 & 0 & 0 \\ 0 & 0 & 0 \\ 0 & 0 & 0\end{array}\right.$

$$
\left.\begin{array}{ccc}
0.135 \Omega^{2} S_{1} & 0.135 \Omega^{2} S_{2} & 0.135 \Omega^{2} S_{3} \\
-0.135 \Omega^{2} C_{1} & -0.135 \Omega^{2} C_{2} & -0.135 \Omega^{2} C_{3} \\
0 & 0 & 0 \\
\hat{S}_{1} & 0 & 0 \\
0 & \hat{S}_{2} & 0 \\
0 & 0 & \hat{S}_{3}
\end{array}\right]
$$

$$
Q_{u}=\left[\begin{array}{cccccc}
1 & 0 & 0 & 0 & 0 & 0 \\
0 & 1 & 0 & 0 & 0 & 0 \\
0 & 0 & 0.299 & 0 & 0 & 0 \\
0 & 0 & 0 & 0.299 & 0 & 0 \\
0 & 0 & 0 & 0 & 0.299 & 0 \\
0 & 0 & 0 & 0 & 0 & 0.299
\end{array}\right],
$$

where $S_{k}=\sin (\omega t+\pi k / 2), \quad C_{k}=\cos (\omega t+\pi k / 2)$ and $\hat{S}_{k}=1961+0.102 \omega^{2}-16.85 S_{k}$, with $\omega=2 \pi / T=10 \pi$ $\mathrm{rad} / \mathrm{s}$, and $k=0,1,2,3$. The $Q_{u}$ matrix above considers the actuators are located in the middle of the blade. These matrices are obtained from the system matrices given in the appendix of Christensen (2004) after removing the second mode of the blades, i.e., after removing the 4th, 6 th, 8 th, 10th lines and columns. Thus, considering only the first mode of the blades.

\section{References}

Abou-Kandil H, Freiling G, Ionescu V and Jank G (2003) Matrix Riccati Equations in Control and Systems Theory. Basel, Switzerland: Birkhäuser.

Anderson BDO (1966) Solution of quadratic matrix equations. Electronics Letters 2(10): 371-372.

Anderson BDO and Moore JB (1990) Optimal Control - Linear Quadratic Methods. Upper Saddle River, USA: Prentice-Hall. Arcara P, Bittanti S and Lovera M (2000) Periodic control of helicopter rotors for attenuation of vibrations in forward flight. 
IEEE Transactions on Control Systems Technology 8(6): 883894.

Athans M (1971) The role and use of the stochastic linear-quadraticGaussian problem in control system design. IEEE Transactions on Automatic Control 16(6): 529-552.

Bekir E and Bucy RS (1976) Periodic equilibria for matrix Riccati equations. Stochastics 2: 1-104.

Bittanti S, Colaneri P and Guardabassi G (1986) Analysis of the periodic Lyapunov and Riccati equations via canonical decomposition. SIAM Journal on Control and Optimization 24(6): 1138-1149.

Bittanti S, Colaneri P and Nicolao GD (1990) Periodic regulators with incomplete and noisy measurements. In: Proceedings of the 29th IEEE Conference on Decision and Control. Honolulu, Hi, pp. 3648-3649.

Bittanti S, Colaneri P and Nicolao GD (1991a) The periodic Riccati equation. In: Bittanti et al. (1991b), pp. 127-162.

Bittanti S, Laub AJ and Willems JC (eds.) (1991b) The Riccati Equation. New York, USA: Springer-Verlag.

Brockett RW (1970) Finite Dimensional Linear Systems. New York, USA: John Wiley \& Sons.

Casti J (1980) The linear-quadratic control problem: Some recent results and outstanding problems. SIAM Review 22(4): 459485.

Christensen RH (2004) Active Vibration Control of Rotor-Blade Systems, Theory and Experiment. $\mathrm{PhD}$ Thesis, Dept. of Mechanical Engineering, Technical University of Denmark, Kgs. Lyngby, Denmark.

Christensen RH and Santos IF (2005) Design of active controlled rotor-blade systems based on time-variant modal analysis. Journal of Sound and Vibration 280(3): 863-882.

Christensen RH and Santos IF (2006) Active rotor-blade vibration control using shaft-based electromagnetic actuation. ASME Journal of Engineering for Gas Turbines and Powerd 128(3): 644-653.

Firoozian R and Stanway R (1988) Active vibration control of turbomachinery: A numerical investigation of modal controllers. Mechanical Systems and Signal Processing 2(3): 243-264.

Fitzgerald B, Sarkar S and Staino A (2018) Improved reliability of wind turbine towers with active tuned mass dampers (ATMDs). Journal of Sound and Vibration 419: 103-122.

Green M and Limebeer DJN (1995) Linear Robust Control. Princeton, USA: Prentice-Hall.

Hairer E, Lubich C and Wanner G (2006) Geometric Numerical Integration: Structure-Preserving Algorithms for Ordinary Differential Equations. Computational Mathematics, 2nd edition. New York, USA: Springer-Verlag.

Hale JK (1969) Ordinary Differential Equations. New York, USA: John Wiley \& Sons.

Hartman P (2002) Ordinary Differential Equations. Classics in Applied Mathematics, 2nd edition. Philadelphia, USA: SIAM.
Harvey CA and Stein G (1978) Quadratic weights for asymptotic regulator properties. IEEE Transactions on Automatic Control 23(3): 378-387.

Hench JJ, Kenney CS and Laub AJ (1994) Methods for the numerical integration of Hamiltonian systems. Circuits, Systems, and Signal Processing 13(6): 695-732.

Hewer GA (1975) Periodicity, detectability and the matrix Riccati equation. SIAM Journal on Control and Optimization 13(6): 1235-1251.

Jakobsen CS, Camino JF and Santos IF (2013) Rotor-blade vibration control using a periodic LQR controller. In: Proceedings of the 2013 SBAI/DINCON. Fortaleza, CE, Brazil, pp. 1-07.

Kalman RE (1960) Contribution to the theory of optimal control. In: Bol. Soc. Mat. Mexicana, volume 5. pp. 102-119.

Kalman RE and Bucy RS (1961) New results in linear filtering and prediction theory. ASME, Journal of Basic Engineering 16(6): 95-108.

Kano H and Nishimura T (1979) Periodic solutions of matrix Riccati equations with detectability and stabilizability. International Journal of Control 29(3): 471-487.

Kenney CS and Lepnik RB (1985) Numerical integration of the differential matrix Riccati equation. IEEE Transactions on Automatic Control 30(10): 962-970.

Khulief YA (2001) Vibration suppression in rotating beams using active modal control. Journal of Sound and Vibration 242(4): 681-699.

Kučera V (1972) A contribution to matrix quadratic equations. IEEE Transactions on Automatic Control 17(3): 344-347.

Lancaster P and Rodman L (1995) Algebraic Riccati Equations. New York, USA: Oxford University Press.

Laub AJ (1979) A schur method for solving algebraic Riccati equations. IEEE Transactions on Automatic Control 24(6): 913-921.

Laub AJ (1991) Invariant subspace methods for the numerical solution of Riccati equations. In: Bittanti et al. (1991b), pp. 163-196.

Pandiyan R and Sinha SC (1999) Periodic flap control of a helicopter blade in forward flight. Journal of Vibration Control 5(5): 7611-777.

Potter JE (1966) Matrix quadratic solutions. SIAM Journal on Applied Mathematics 14(3): 496-501.

Ravi R, Pascoal AM and Khargonekar PP (1990) Normalized coprime factorizations and the graph metric for linear timevarying systems. In: Proceedings of the 29th IEEE Conference on Decision and Control. Honolulu. Hawaii, pp. 1241-1246.

Reid WT (1972) Riccati Differential Equations, Mathematics in Science and Engineering, volume 86. San Diego, CA, USA: Academic Press.

Safonov MG and Athans M (1977) Gain and and phase margin for multiloop LQG regulators. IEEE Transactions on Automatic Control 22(2): 173-179. 
Sherrill RE, Sinclair AJ, Sinha SC and Lovell TA (2015) LyapunovFloquet control of satellite relative motion in elliptic orbits. IEEE Transactions on Aerospace and Electronic Systems 51(4): 2800-2810.

Sinha SC and Joseph P (1994) Control of general dynamic systems with periodically varying parameters via LiapunovFloquet transformation. ASME Journal of Dynamic Systems, Measurement, and Control 116(4): 650-658.

Skjoldan PF and Hansen MH (2009) On the similarity of the coleman and lyapunov-floquet transformations for modal analysis of bladed rotor structures. Schock and Vibration 327(3): 424-439.

Szász G and Flowers GT (2000) Vibration suppression in bladed-disk assemblies with deliberate mistuning via magnetic bearings. Journal of Vibration Control 6(6): 903-921.

Szász G and Flowers GT (2001) Time periodic control of a bladed disk assembly using shaft based actuation. ASME Journal of Vibration and Acoustics 123(3): 395-398.

Varga A (2008) On solving periodic Riccati equations. Numerical Linear Algebra with Applications 15: 809-835.

Wiedemann M and Person M (1992) Die stabilität und die empfindlichkeit der stabilität periodisch zeitvarianter systeme in der rotordynamik. Forschung im Ingenieurwesen 58(9): 213-222.

Willems JC and Mitter SK (1971) Controllability, observability, pole allocation, and state reconstruction. IEEE Transactions on Automatic Control 16(6): 582-595.

Wonham WM (1968) On a matrix Riccati equation of stochastic control. SIAM Journal on Control and Optimization 6(4): 681697.

Yakubovich VA and Starzhinskii VM (1975) Linear differential equations with periodic coefficients, volume 1-2. New York, USA: John Wiley \& Sons. 